\title{
Laser cooling of trapped ions: The influence of micromotion
}

\author{
J. I. Cirac \\ Departamento de Fisica Aplicada, Facultad de Quimicas, Universidad de Castilla-La Mancha, 13071 Ciudad Real, Spain \\ L. J. Garay \\ ESA IUE Observatory, P.O. Box 50727, E-28080 Madrid, Spain \\ R. Blatt \\ Institut für Laser-Physik, Jungiusstrasse 9, D-2000, Hamburg 36, Germany
}

A. S. Parkins and P. Zoller

Joint Institute for Laboratory Astrophysics and Department of Physics, University of Colorado, Boulder, Colorado 80309-0440

(Received 8 July 1993)

\begin{abstract}
Laser cooling of a single trapped ion in a Paul trap is discussed theoretically in the LambDicke limit, with full consideration of the time dependence of the trapping potential. Resulting mean kinetic energies are defined as time averages over one period of the micromotion and are compared with final temperatures expected from the laser cooling treatment with harmonic traps. For laser-atom detunings close to the micromotion frequency the results differ significantly from those expected for a harmonic trap potential. A physical interpretation is given and simple formulas are derived for the strong confinement case.
\end{abstract}

PACS number(s): $32.80 . \mathrm{Pj}$

\section{INTRODUCTION}

The ability to cool single trapped ions optically has become an important tool for fundamental experiments both in quantum optics and in precision spectroscopy $[1,2]$. This is due to the fact that a single trapped and cooled ion provides an almost ideal quantum system which can be modeled by quantum optics theory and thus considered for applications with time and frequency standards $[3,4]$.

The standard theory of laser cooling of trapped ions assumes motion of a laser-driven ion in a (static) onedimensional harmonic oscillator trapping potential [5-8]. Almost all experiments with single laser-cooled ions, on the other hand, have been performed with Paul traps where a rf of frequency $\omega$ and a dc electric field are used to generate a trapping potential. Thus the trapping potential of a Paul trap is explicitly time dependent. In general, the ion motion in a Paul trap is governed by a fast oscillation at the driving frequency $\omega$ (micromotion), superimposed on a slow secular motion (macromotion) [1]. To the extent that the frequency of the macromotion is much smaller than that of the micromotion, adiabatic elimination of the fast time scales $1 / \omega$ allows one to describe the ion dynamics as motion in an effective harmonic oscillator potential (pseudopotential). Thus standard laser cooling theory is based on the assumption that the time scale of the rf field is much faster than all other time scales of the problem. In experiments the effects of the micromotion are clearly visible as additional resonances in excitation spectra [9], and it appears necessary to investigate in which way the time dependent trapping field influences the cooling dynamics, the cooling rates, and the final temperatures. It is the purpose of this paper to develop a theory of laser cooling in Paul traps that includes the influence of the micromotion.

A theoretical description of laser cooling of a single two-level ion trapped in a harmonic potential was given some time ago by Wineland and Itano [5] and by Stenholm, Javanainen, and Lindberg [6-8]. They derived simple formulas for both the cooling rate and the final energy reached by the ion at the end of the cooling process. For trap frequencies $\nu$ smaller than the natural linewidth $\Gamma$ of the optical transition used for laser cooling (i.e., the weak confinement or weak-binding limit), the final energy of the ion is limited by $E=\hbar \Gamma / 2$ (Doppler limit). For $\nu>\Gamma$ (i.e., strong confinement or strong-binding limit) the trapped ion develops well-resolved absorption sidebands at the trap frequency and selective absorption on the lower sideband can optically pump the ion to its lowest vibrational state, and therefore to the lowest energy permitted by quantum mechanics $E=\hbar \nu / 2$ (sideband cooling).

When discussing the influence of micromotion, the notion of temperature has to be reconsidered. In a harmonic trap laser cooling theories predict a Boltzmann distribution of the occupation of the harmonic oscillator states which allows the assignment of a temperature. However, with the micromotion present, the Hamiltonian that describes the motion of the ion in the trap is time dependent and therefore the concept of time-independent eigenstates of the trap Hamiltonian fails. Nevertheless, it is always possible to define the kinetic energy via the expectation value of the squared momentum $\left\langle P(t)^{2}\right\rangle$ which is explicitly time dependent. For a comparison with experimental results we define as the mean kinetic energy 
a time-averaged value $\overline{\left\langle P^{2}\right\rangle} /(2 m)$ where the time average is taken over one period $2 \pi / \omega$ of the micromotion [11]. As we will show below, this mean kinetic energy can be compared to the kinetic energy (as given by the temperature) obtained for the harmonic pseudopotential approximation and thus one can study the influence of the micromotion on the dynamics of laser cooling of a single ion in Paul traps. Although in this paper we concentrate on two-level ions, the general features of the results are valid and applicable to laser cooling processes for multilevel ions in a time-dependent potential.

From our results, it turns out that the treatment of laser cooling with harmonic traps describes the cooling dynamics sufficiently well as long as the laser-atom detuning $\delta$ is small compared to the micromotion frequency $\omega$, more precisely, for $|\delta|<\omega-\nu-\Gamma$. We will show that when this condition is not fulfilled, cooling may arise for additional detunings and heating may appear where cooling was expected. It will also be shown that laser cooling is possible for detunings even above resonance, i.e., for blue detunings, using both traveling- and standing-wave laser fields.

This paper is organized as follows: in Sec. II an overview of the most important features is presented, with an emphasis on the phenomena that arise when taking full account of the micromotion in a Paul trap. The theoretical model is introduced in Sec. III. In Sec. IV we give a physical description of the cooling process, deriving a simple formula for the final kinetic energy in the strong confinement limit. In Sec. $\mathrm{V}$ we describe a method that permits us to numerically evaluate the kinetic energy in terms of matrix continued fractions and we derive some analytical results pertaining to the role of micromotion in the stationary state. A more detailed discussion of the results is presented in Sec. VI. Finally, some technical details of the calculations are given in Appendixes A and B.

\section{PRELIMINARY CONSIDERATIONS}

In the following we will consider laser cooling of a single trapped ion confined in a Paul trap including the full time dependence of the trapping potential. With the assumption that the ion is confined to spatial dimensions smaller than the optical wavelength (Lamb-Dicke limit), we have obtained the stationary behavior of the ion. In this section we give a qualitative overview of the main results presented in this paper.

The motion of an ion in a Paul trap is described by the Hamiltonian

$$
H_{t p}=\frac{P^{2}}{2 m}+\frac{1}{2} m W(t) X^{2}
$$

where

$$
W(t)=\frac{1}{4} \omega^{2}[a-2 q \cos (\omega t)] .
$$

Here $P^{2} / 2 m$ denotes the kinetic energy of the ion with mass $m$. The parameter $a$ is proportional to the dc volt- age whereas $q$ is related to an ac voltage of frequency $\omega$ applied to the trap electrodes.

We begin our discussion by describing the motion of the ion from the classical point of view. Starting from the Hamiltonian (2.1) one can derive the equation of motion for $X(t)$, which is the Mathieu equation

$$
\ddot{X}(t)+\frac{\omega^{2}}{4}[a-2 q \cos (\omega t)] X(t)=0 .
$$

As it is well known, stable ion trapping is only possible for certain values of the $(a, q)$ parameters, and is usually described in terms of a stability chart [12]. A stable solution of this equation can be expressed as

$$
X(t)=A F(t)+\text { c.c. },
$$

where the complex parameter $A$ depends on the initial conditions $X(0)$ and $P(0)$, and

$$
F(t)=\sum_{n=-\infty}^{\infty} c_{2 n} e^{i(\nu+n \omega) t} .
$$

The frequency $\nu$ is a function of $a, q$, and $\omega$ (more specifically, $2 \nu / \omega$ is a function of $a$ and $q$ ). Assuming $c_{0}=1$, we can write $c_{2 n}(n \neq 0)$ in terms of a continued fraction [13]

$$
\begin{aligned}
c_{0} & =1 \\
c_{ \pm 2 n} & =\frac{c_{( \pm 2 n \mp 2)}}{V_{ \pm 2 n}-\frac{1}{V_{( \pm 2 n \pm 2)}-1}},
\end{aligned}
$$

where

$$
V_{2 n}=\frac{a-(2 \nu / \omega+2 n)^{2}}{q} .
$$

From Eq. (2.6) it can be shown that, in the limit $a, q \ll 1$, the inequality $c_{0}=1 \gg\left|c_{ \pm 2}\right|$ holds, and therefore, the motion is essentially governed by a secular motion with frequency $\nu \simeq \frac{1}{2} \omega\left(a+q^{2} / 2\right)^{1 / 2}(\ll \omega)$. Superimposed on this macromotion is a small-amplitude modulation with the micromotion frequency $\omega$. In this case the pseudopotential approximation applies, which amounts to expanding all the variables in terms of $q$ and $a$, and retaining only the lowest order terms in these expansions. In this limit, $X(t)$ can be approximated by

$$
X(t) \simeq A e^{i \nu t}+\text { c.c. },
$$

that is, one can consider that the ion moves in an effective harmonic potential of frequency $\nu$, without taking into account the micromotion. However, to evaluate the kinetic energy one has to consider the micromotion, since despite the fact that the amplitudes of the micromotion oscillations are small, their frequency is much larger than $\nu$, i.e., it contributes to the total kinetic energy. Within the framework of the pseudopotential approximation (and for $q>a$ ) the kinetic energy due to micromotion is reinterpreted as the potential energy in the effective harmonic trap, which allows us to restrict the study of the motion in Paul traps to harmonic traps. 
This approximation is also used in all theories concerning laser cooling of ions in Paul traps $[15,16]$. Hence, all theories treating laser cooling of ions in a Paul trap merely consider the secular motion of an ion in the effective harmonic trap and neglect the effects of the micromotion other than a contribution to the kinetic energy of the same order as that of the secular motion. This leads one to consider the micromotion as a source of further oscillatory motion, which in fact leads to the appearance of sidebands as, e.g., observed in absorption spectra [9]. Qualitatively, we may expect that the presence of the micromotion affects the cooling dynamics and it is then worthwhile investigating whether or not it could be used for additional cooling.

In order to study the role played by micromotion in laser cooling let us first give a brief summary of the theoretical description of laser cooling in harmonic traps. The energy of an ion moving in a harmonic potential of frequency $\nu$ is given by

$$
E=\frac{\left\langle P^{2}\right\rangle}{2 m}+\frac{1}{2} m \nu^{2}\left\langle X^{2}\right\rangle,
$$

where $\langle\cdots\rangle$ stands for quantum expectation value. This energy can be calculated in the Lamb-Dicke limit [10], and therefore the kinetic energy of a laser cooled ion in a stationary state can be readily derived. For two-level ions interacting with a laser traveling wave, cooling occurs for negative laser-ion detunings $(\delta<0)$, and the minimum energies are found for low Rabi frequencies [6]. In a standing wave, however, the situation is different, since the ion can be localized at any position with respect to the standing wave [10]. So, at the node the final energy does not depend on the Rabi frequency, and it is a factor of about 2 smaller than in the traveling case. At the point of maximum gradient and for high Rabi frequencies laser cooling for positive detunings $(\delta>0)$ is possible.

The pseudopotential approximation also allows a simple explanation of cooling by transforming to a frame which is moving with the trapped ion. In this frame the ion senses, in addition to the laser at frequency $\omega_{L}$, two motional sidebands $\omega_{L} \pm \nu$ which arise from the harmonic motion of the ion in the trap with frequency $\nu$ [compare Eq. (2.8)]. Higher order sidebands are neglected in the Lamb-Dicke limit. It can be shown that any absorption of a photon of frequency $\omega_{L}+\nu\left(\omega_{L}-\nu\right)$ is accompanied by a decreasing (increasing) of the ion's energy [10]. Cooling is achieved when the relative absorption on these frequencies is such that the energy of the ion is reduced on the average (i.e., when the ion is more likely to absorb photons at $\left.\omega_{L}+\nu\right)$. Laser cooling is particularly efficient when the secular frequencies are larger than the natural linewidth $\Gamma$ of the cooling transition; in this case, by choosing the laser frequency in such a way that the sideband at $\omega_{L}+\nu$ is on resonance with the two-level transition, we obtain the most efficient cooling mechanism and the ion ends up in its lowest vibrational state (sideband cooling).

In all experimental realizations and measurements, such as those used for precision spectroscopy, one is interested in the ion kinetic energy achieved after laser cooling the ion, since this is the quantity that eventually limits the precision of a measurement. As will be shown in Sec. $\mathrm{V}$ below, in the Lamb-Dicke limit, the kinetic energy and spatial variance of a single trapped ion can be calculated under the influence of laser cooling, taking also into full account the time dependence of the potential. The kinetic energy is determined by the expectation value of the squared momentum $\left\langle P(t)^{2}\right\rangle$ which, even in the stationary state, oscillates according to the different modes given in Eq. (2.4). Hence we take a time average over one period of frequency $\omega$ to determine the mean kinetic energy $\overline{\left\langle P^{2}\right\rangle} /(2 m)$ and the mean variance of the spatial extension $\overline{\left\langle X^{2}\right\rangle}$. In addition, we can determine the maximum and minimum kinetic energy and spatial extension that are attained in one micromotion period and compare these to values predicted by the pseudopotential approximation (i.e., the harmonic trap approximation).

As stated above, in the absence of cooling the harmonic trap is recovered in the limit $a, q \rightarrow 0$ (and $\omega \rightarrow \infty$, such that $\nu$ remains constant). We have found that this is also true even in the presence of cooling. In particular, for $a=0$ we find

$$
\begin{aligned}
& \overline{\left\langle P^{2}\right\rangle} \longrightarrow 2\left\langle P^{2}\right\rangle_{h t}, \\
& \overline{\left\langle X^{2}\right\rangle} \longrightarrow\left\langle X^{2}\right\rangle_{h t},
\end{aligned}
$$
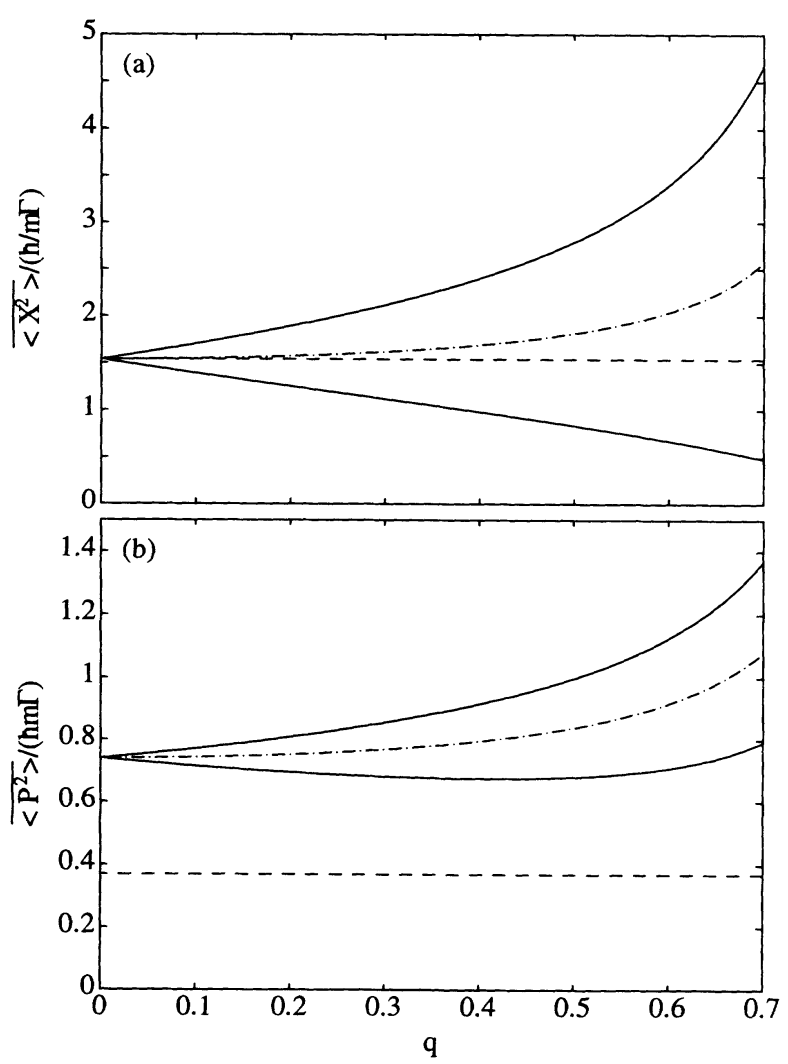

FIG. 1. Kinetic energy (a) and position variance (b) as functions of the Mathieu $q$ parameter for an ion at the node of a standing wave. Parameters are $a=0, \nu=0.2 \Gamma$, and $\Delta=-\Gamma / 2$. Solid lines give the minimum and maximum values in one micromotion period; dot-dashed lines give the mean values and the dashed lines show the results calculated with a harmonic trap. Note that the stability boundary for the present case is $q \approx 1$. 


$$
\frac{\left\langle P^{2}\right\rangle_{h t}}{2 m}=\frac{1}{2} m \nu^{2}\left\langle X^{2}\right\rangle_{h t}
$$

and the amplitude of the micromotion tends to zero [the subscript $h t$ indicates the value obtained using the (effective) harmonic oscillator trap]. This behavior is illustrated in Figs. 1(a) and 1(b) where the time averaged position variance $\overline{\left\langle X^{2}\right\rangle}$ and kinetic energy $\overline{\left\langle P^{2}\right\rangle} / 2 m$ (in units of $\hbar \Gamma / 2)$ are plotted as a function of the parameter $q$ (dashed-dotted lines). We have assumed that the ion is located at the node of a standing laser wave. In these figures we have also indicated the maximum and minimum values of the position variance and kinetic energy during one micromotion period (upper and lower solid lines, respectively) and the corresponding values expected from the harmonic trap approximation (dashed lines). The curves of Fig. 1 were obtained from the cooling theory derived in the following sections.

Figure 2 shows $\overline{\left\langle P^{2}\right\rangle}$ and $\overline{\left\langle X^{2}\right\rangle}$ for a trap frequency $\nu=1.5 \Gamma$, and a micromotion frequency $\omega=10 \Gamma$ as a function of the detuning for the case of an ion at the node of a standing-wave field. The solid lines represent the results of our theory (Sec. V), and the dashed lines show the detuning dependence for a harmonic potential. For the parameters in this figure we have sideband cooling for the harmonic trap: for the optimum detuning of $\Delta=-\nu$ we find an energy close to $E=\hbar \nu / 2$, or
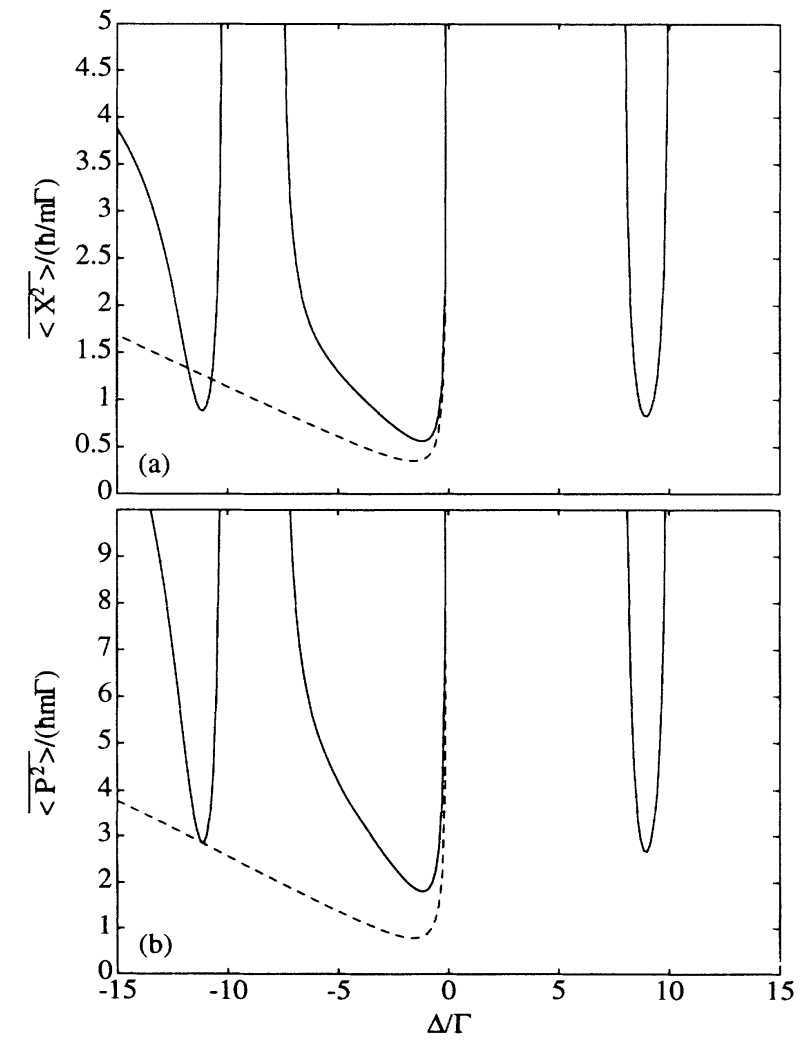

FIG. 2. Mean position variance (a) and mean kinetic energy (b) as a function of the detuning $\Delta$ for an ion at the node of a standing wave. Parameters are $a=0, \nu=1.5 \Gamma$, and $\omega=10$. The dashed lines show the results calculated with a harmonic trap. $\left\langle P^{2}\right\rangle / 2 m=\hbar \nu / 4=3 / 8 \hbar \Gamma$ [Fig. $\left.2(\mathrm{~b})\right]$. Note first that in Fig. 2 the regions where cooling and heating are observed differ considerably when micromotion is considered. As will be shown below, this qualitative behavior is general and remains unchanged when we consider other locations of the ion in the standing-wave or in traveling-wave configurations [see Figs. 3(a) and 3(b)]. In addition, we find increased cooling for detunings below the resonance (red detunings) and there are resonances where heating is observed (when the laser detuning is close to the rf frequency) and, more surprisingly, there is cooling observed for blue detunings. The minimum energy calculated in the harmonic trap approximation is no longer achieved, and more importantly, a minimum energy may be found now for much larger detunings with values even below that predicted for a harmonic trap.

This behavior may be understood in a qualitative way by transforming to a frame that is moving with the ion. In this frame the ion appears at rest and interacts with the laser and the motional sidebands: in the Lamb-Dicke limit the ion senses light waves of frequencies $\omega_{L}, \omega_{L} \pm \nu$, $\omega_{L} \pm(\nu \pm \omega)$, etc. [compare (2.4) with (2.8)]. These frequencies are represented in Fig. 4, where their intensities are indicated by the height of the vertical bars which, in turn, are determined by the coefficients $c_{2 n}$ given in Eq. (2.6). Absorption of photons of frequencies $\omega_{L}+n \omega+\nu(n=0, \pm 1, \ldots)$ is accompanied by a de-

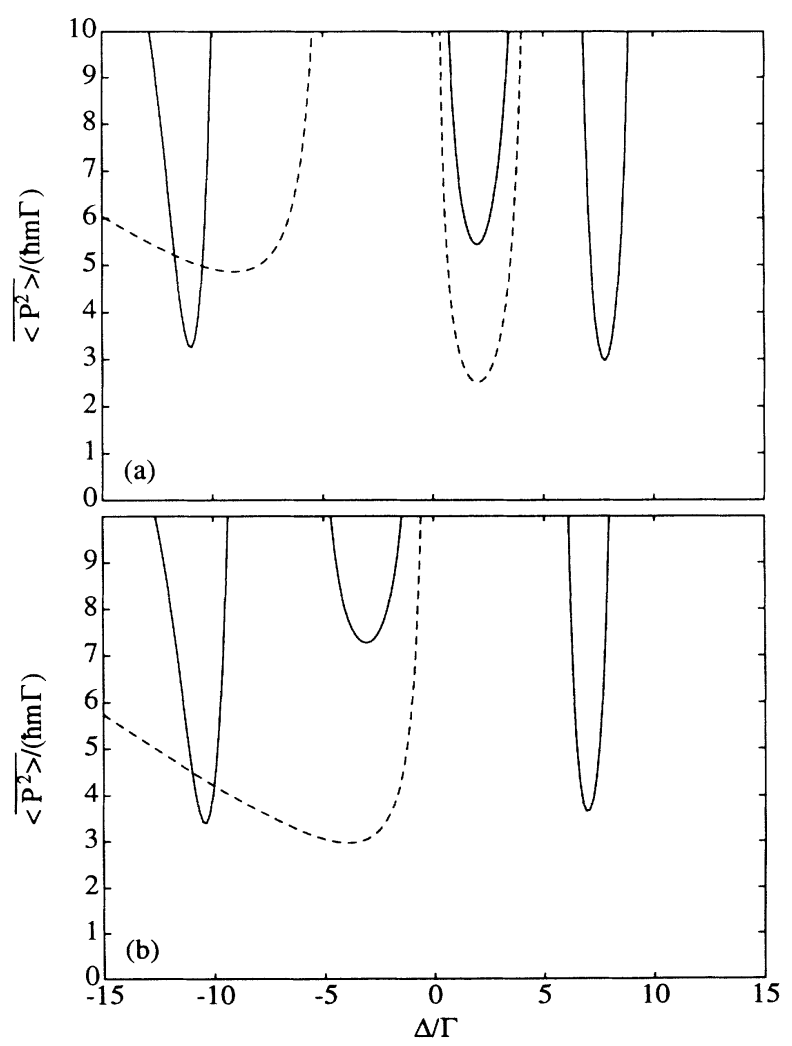

FIG. 3. Mean kinetic energy as a function of the detuning for a two-level ion (a) located at the point of maximum gradient in a standing wave, with $\Omega=5 \Gamma$; (b) in a traveling wave, with $\Omega=2 \Gamma$. Other parameters are $a=0, \nu=1.5 \Gamma$, $\omega=10 \Gamma$, and $k=0.01(m=1)$. 


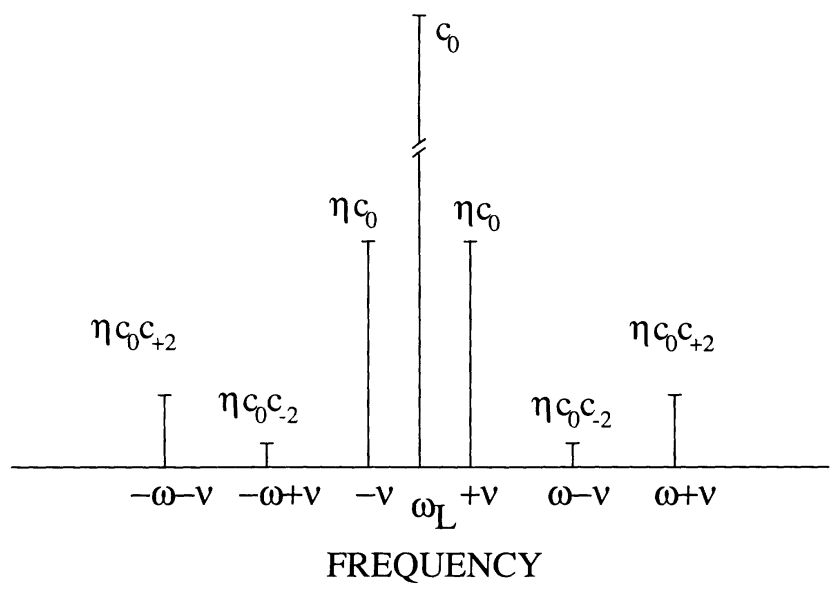

FIG. 4. Schematic representation of the lasers seen in a frame moving with the ion. Note that the sidebands are a factor of $\eta=k /\left(\xi 2^{1 / 2}\right) \ll 1$ times smaller than the central laser.

crease of the kinetic energy of the ion, while absorption of laser photons at $\omega_{L}+n \omega-\nu(n=0, \pm 1, \ldots)$ increases the kinetic energy. Hence, as in the case of the cooling theory for the harmonic trap, the absorption at different laser-atom detunings determines the cooling. This argument is particularly simple when the frequencies sensed by the ion are well separated, i.e., in the sideband limit $\Gamma \ll \nu \ll \omega-\Gamma$. In this case, for a detuning close to $\Delta=-(\nu+n \omega)(n=0, \pm 1, \ldots)$, the ion preferentially absorbs photons of frequency $\omega_{L}+\nu+n \omega$ and-as in the case of sideband cooling in harmonic traps - the ion is cooled very efficiently. As we will show in Sec. IV, this simple picture permits us to calculate the final kinetic energy for any values of $a$ and $q$. On the other hand, heating appears for detunings around $\Delta=\nu \pm n \omega$. When for a given detuning the "lasers" sensed by the ion are not so well separated $(\Gamma>\nu$ or $\Gamma>\omega-\nu)$, the ion can absorb photons simultaneously from more than one light wavelength; this treatment of laser cooling of ions becomes increasingly complicated, and more difficult to interpret, although it can still be understood qualitatively in these terms. Note finally that for $q \ll 1$ we have that $c_{ \pm 2} \propto q \ll 1$, i.e., the lasers of frequencies $\omega_{L}$ and $\omega_{L} \pm \nu$ are much stronger than the others (see Fig. 4). Hence, for detunings $|\delta|<\omega-\nu-\Gamma$ the ion only interacts with these three frequencies, and the resulting problem is formally the same as for a harmonic trap (see Sec. IV).

\section{MODEL}

We consider a two-level ion trapped in a onedimensional time-dependent potential. The ion interacts with a laser field and is damped by spontaneous emission. In a frame rotating at the laser frequency $\omega_{L}$, the master equation for the system has the form (we choose units with $\hbar=1$ and mass $m=1$ in the following)

$$
\dot{\rho}=-i\left[H_{t p}+H_{I}+V_{d i p}, \rho\right]+\mathcal{L}^{d} \rho,
$$

where $H_{t p}$ is the time-dependent Hamiltonian for the external degrees of freedom given in (2.1),

$$
H_{I}=-\frac{1}{2} \Delta \sigma_{z}
$$

is the free Hamiltonian for the internal energy levels of the ion, and

$$
V_{d i p}(X)=\frac{\Omega}{2}\left[f(X) \sigma^{+}+f(X)^{*} \sigma^{-}\right]
$$

denotes the dipole interaction between the laser and the internal degrees of freedom of the ion. Here, $\sigma_{ \pm, z}$ are the usual transition operators describing the two-level transition, $\Delta=\omega_{L}-\omega_{0}$ is the laser-ion detuning, and the form of the function $f(X)$ depends on whether we are considering a standing-wave or a traveling-wave laser field, namely

$$
f(X)=\cos (k X+\phi)
$$

and

$$
f(X)=e^{-i k X},
$$

respectively. Spontaneous emission is described by the dissipative Liouville term

$$
\mathcal{L}^{d} \rho=\frac{\Gamma}{2}\left(2 \sigma^{-} \tilde{\rho} \sigma^{+}-\sigma^{+} \sigma^{-} \rho-\rho \sigma^{+} \sigma^{-}\right),
$$

where $\Gamma$ is the spontaneous emission rate, and

$$
\tilde{\rho}=\frac{1}{2} \int_{-1}^{1} d z W(z) e^{i k X z} \rho e^{-i k X z}
$$

with $W(z)$ being the angular distribution of spontaneous emission which, for usual dipole transitions, is $W(z)=$ $\frac{3}{4}\left(1+z^{2}\right)$.

In the Lamb-Dicke limit, we can expand master equation (3.1) up to second order in $k X$, obtaining

$$
\begin{aligned}
V_{d i p}(X)= & \frac{\Omega}{2}\left[f(0) \sigma^{+}+f(0)^{*} \sigma^{-}\right] \\
& +X\left[f^{\prime}(0) \sigma^{+}+f^{\prime}(0)^{*} \sigma^{-}\right] \\
& +\frac{1}{2} X^{2}\left[f^{\prime \prime}(0) \sigma^{+}+f^{\prime \prime}(0)^{*} \sigma^{-}\right], \\
\mathcal{L}^{d} \rho= & \frac{\Gamma}{2}\left(2 \sigma^{-} \rho \sigma^{+}-\sigma^{+} \sigma^{-} \rho-\rho \sigma^{+} \sigma^{-}\right) \\
& +\alpha \Gamma k^{2} \sigma^{-}\left(2 X \rho X-X^{2} \rho-\rho X^{2}\right) \sigma^{+},
\end{aligned}
$$

where the angular distribution $W(z)$ for spontaneous emission is normalized, and an even function of $z$. Furthermore, we have defined

$$
\alpha=\frac{1}{2} \int_{-1}^{1} d z z^{2} W(z)
$$

which equals $2 / 5$ for usual dipole transitions. In all these expressions the prime stands for "spatial" derivative.

The master equation (3.1) with $V_{d i p}$ and $\mathcal{L}^{d}$ given in 
(3.8) is the starting point for the analysis presented in the following sections.

\section{LASER COOLING IN A PAUL TRAP}

We next present an approach to the problem which permits us to understand the cooling process in terms of the interaction between the ion and the different lasers "sensed" by the ion due to its motion. Within this approach we will derive a simple formula for the kinetic energy reached by the ion in the stationary state, valid in the sideband limit. We start by considering the Hamiltonian part of the interaction up to first order in the Lamb-Dicke expansion,

$$
\begin{aligned}
H= & H_{t p}(t)+\frac{1}{2} \omega_{0} \sigma_{z}+\frac{\Omega}{2} f(0)\left(\sigma^{+} e^{-i \omega_{L} t}+\text { H.c. }\right) \\
& +\frac{\Omega}{2}\left[f^{\prime}(0) \sigma^{+} e^{-i \omega_{L} t}+\text { H.c. }\right] X .
\end{aligned}
$$

Now we look at the frame moving with the ion, which is defined by the unitary operator that fulfills

$$
\dot{U}(t)=-i H_{t p}(t) U(t)
$$

In this new picture the states evolve with the Hamiltonian

$$
\begin{aligned}
\tilde{H}= & \frac{1}{2} \omega_{0} \sigma_{z}+\frac{\Omega}{2} f(0)\left(\sigma^{+} e^{-i \omega_{L} t}+\text { H.c. }\right) \\
& +\frac{\Omega}{2}\left[f^{\prime}(0)\left(\sigma^{+} e^{-i \omega_{L} t}+\text { H.c. }\right)\right] \tilde{X}(t),
\end{aligned}
$$

while the external operators evolve with the Hamiltonian $\tilde{H}_{t p}=U^{\dagger} H_{t p} U$. Consequently, the operator $\tilde{X}(t)=$ $U^{\dagger} X(t) U$ appearing in (4.3) satisfies the quantum Mathieu equation. This equation has been extensively studied, for example, by Glauber [14] (see also Appendix A). In particular one can write the evolution of $\tilde{X}(t)$ in terms of two operators $A$ and $A^{\dagger}$, which fulfill the commutation relation $\left[A, A^{\dagger}\right]=1$. As in the classical case, $A$ and $A^{\dagger}$ depend on the initial conditions $X(0)$ and $P(0)$. Inserting the expression (A2) for $\tilde{X}(t)$ in (4.3) we can write the Hamiltonian $\tilde{H}$ as

$\tilde{H}=\frac{1}{2} \omega_{0} \sigma_{z}+\frac{\Omega}{2} f(0)\left(\sigma^{+} e^{-i \omega_{L} t}+\right.$ H.c. $)+\left[\sum_{n=-\infty}^{\infty} \frac{\Omega}{2} \frac{f^{\prime}(0)}{\sqrt{2} \xi} c_{2 n} \sigma^{+}\left[A e^{-i\left(\omega_{L}+\nu+n \omega\right) t}+A^{\dagger} e^{-i\left(\omega_{L}-\nu-n \omega\right) t}\right]+\right.$ H.c. $]$

where $\xi$ has been defined in Appendix A. The interpretation of this Hamiltonian is straightforward. In the new interaction picture (in the moving frame), the ion senses: (i) a (strong) laser of frequency $\omega_{L}$, with Rabi frequency equal to $\Omega f(0)$; (ii) pairs of lasers of frequencies $\omega_{L} \pm(\nu+n \omega)(n=0, \pm 1, \ldots)$ and corresponding Rabi frequencies of $\Omega c_{2 n} f^{\prime}(0) /(\sqrt{2} \xi)$. Note first that these last frequency components are weak compared to the first ones, since they give the first-order contribution in the Lamb-Dicke limit to the Hamiltonian $\tilde{H}$. Note also that for $n>0(n<0)$ in general $\left|c_{2 n}\right|<\left|c_{2(n-1)}\right|\left(\left|c_{2 n}\right|>\left|c_{2(n-1)}\right|\right)$ [13], and therefore the intensity of the sidebands decreases as $|n|$ increases (see Fig. 4).

The fact that the ion can absorb more efficiently one of the sideband frequencies determines the cooling process. In order to describe laser cooling let us first consider the case in which the transition frequency is close to one of these laser frequencies $\omega_{0} \simeq \omega_{L}+(\nu+n \omega)$. Then, this laser is more likely to be absorbed than the others, provided that the transition linewidth $\Gamma$ is smaller than the frequency difference between the remaining lasers and the transition frequency, i.e., for

$$
\omega-\Gamma \gg \nu \gg \Gamma \text {. }
$$

In this case, the remaining sideband frequencies can be ignored, and the Hamiltonian reduces to

$$
\tilde{H} \simeq \frac{1}{2} \omega_{0} \sigma_{z}+\left[\frac{\Omega}{2} \frac{f^{\prime}(0)}{\sqrt{2} \xi} c_{2 n} \sigma^{+} A e^{-i\left(\omega_{L}+\nu+n \omega\right) t}+\text { H.c. }\right] .
$$

Moving now to a second interaction picture defined by the unitary operator

$$
V=e^{-\frac{1}{2} i \omega_{L} \sigma_{z}} e^{i(\nu+n \omega) A^{\dagger} A},
$$

this Hamiltonian becomes

$$
\hat{H} \simeq-\frac{1}{2} \delta \sigma_{z}+(\nu+n \omega) A^{\dagger} A+\left[\frac{\Omega}{2} \frac{f^{\prime}(0)}{\sqrt{2} \xi} c_{2 n} \sigma^{+} A+\text { H.c. }\right] .
$$

Note that this effective Hamiltonian is time independent. This makes it possible to explain the cooling dynamics via the eigenstates of $A^{\dagger} A$, i.e., the states $|N\rangle$ defined in Appendix A. In terms of these states, each absorption of a laser photon by the ion is accompanied by a decreasing of $N$ in one unit. A subsequent spontaneous decay leaves the two-level ion in its ground state, ready for the next absorption. After several absorption-emission cycles, the ion ends up in its ground state and with $N=0$. This is in clear analogy to what occurs in cavity quantum electrodynamics. In fact $\hat{H}$ is the so-called Jaynes-Cummings Hamiltonian and, up to first order in the Lamb-Dicke expansion, the problem treated here coincides with the interaction of a two-level atom with a cavity mode, including spontaneous emission. As is well known, in this case the atom ends up in its ground state and the cavity mode in the vacuum state.

This cooling mechanism is based on the same principle as the so-called sideband cooling for harmonic traps. The only difference is that, in the presence of micromotion, one can perform sideband cooling with all the cooling 
lasers sensed by the ion, that is, for detunings

$$
\Delta=-(\nu+n \omega)(n=0, \pm 1, \ldots)
$$

At the end of the cooling process we have that $\left\langle A^{\dagger} A\right\rangle=$ $\left\langle A^{2}\right\rangle=\left\langle A^{\dagger 2}\right\rangle=0$, so that the expected value of $P(t)^{2}$ results in

$$
\left\langle P(t)^{2}\right\rangle=\frac{1}{2 \xi^{2}}|\dot{F}(t)|^{2},
$$

as can be deduced from Eq. (A5b). Averaging this expression over one micromotion period we obtain

$$
\overline{\left\langle P^{2}\right\rangle}=\frac{1}{2 \xi^{2}} \sum_{n=-\infty}^{\infty}\left|c_{2 n}\right|^{2}|\nu+n \omega|^{2} .
$$

Analogously, one can show that, in this case,

$$
\left\langle X(t)^{2}\right\rangle=\frac{1}{2 \xi^{2}}|F(t)|^{2},
$$

and consequently

$$
\overline{\left\langle X^{2}\right\rangle}=\frac{1}{2 \xi^{2}} \sum_{n=-\infty}^{\infty}\left|c_{2 n}\right|^{2} .
$$

All these expressions can be simply evaluated, and only depend on the Mathieu parameters $a, q$, and $\omega$. On the other hand, it can be easily shown that in the pseudopotential limit $q \ll 1$ (and for $a=0$ ), when $\delta=-\nu$, expressions (4.11) and (4.13) reduce to $\overline{P^{2}}=2 \overline{X^{2}}=\nu$, which exactly coincides with the final energy for the harmonic trap case when sideband cooling applies. This is in agreement with what we already advanced in Sec. II [Eqs. (2.10)].

If we now consider the case in which the transition frequency is close to one of the laser frequencies $\omega_{0} \simeq$ $\omega_{L}-(\nu+n \omega)$, the effective Hamiltonian takes the form

$\hat{H} \simeq-\frac{1}{2} \delta \sigma_{z}+(\nu+n \omega) A^{\dagger} A+\left[\frac{\Omega}{2} \frac{f^{\prime}(0)}{\sqrt{2} \xi} c_{2 n} \sigma^{+} A^{\dagger}+\right.$ h.c. $]$.

The arguments given above also apply, but the absorption of a laser photon is now accompanied by an increase in the quantum number $N$. As a result, the ion is heated.

To summarize, when condition (4.5) is satisfied, i.e., the lasers seen by the ion are well separated, absorption of a laser photon of frequency $\omega_{L}+\nu+n \omega$ leads to cooling, while absorption of a laser photon of frequency $\omega_{L}-(\nu+n \omega)$ leads to heating. If condition (4.5) is not fulfilled, laser cooling can be also understood in terms of absorption of photons from these lasers, although the dynamics is more involved since the ion can sense various lasers at the same time.

\section{SOLUTION TO THE MASTER EQUATION}

For harmonic traps, master equation (3.1) can be transformed into an infinite set of ordinary differential equations either by projecting it onto eigenstates of the harmonic oscillator Hamiltonian, or by deriving the evolution equations for the expectation values of products of annihilation, creation, and atomic operators. These equations can be subsequently solved by numerical methods. This is no longer possible for the time-dependent Hamiltonian considered here. Instead, one can use the position and momentum operators themselves rather than the ill-defined annihilation and creation operators.

We are particularly interested in the equations for the expectation values $\left\langle X^{2}\right\rangle$ and $\left\langle P^{2}\right\rangle$ up to second order in the Lamb-Dicke expansion. In view of (3.8), they are connected in first and second order to other quantities such as $\left\langle X \sigma_{ \pm, z}\right\rangle$ and as $\left\langle X^{2} \sigma_{ \pm, \sigma_{z}}\right\rangle$, respectively. Consequently we also have to include the equations for the former up to first order, and for the latter, up to zeroth order. In zeroth order the internal and external degrees of freedom are decoupled (note that they are connected by $V_{\text {dip }}$ and $\mathcal{L}^{d}$, which in lowest order do not depend on $X)$. Hence we can factorize

$$
\begin{aligned}
\left\langle X^{2} \sigma_{ \pm, z}\right\rangle & =\left\langle X^{2}\right\rangle\left\langle\sigma_{ \pm, z}\right\rangle \\
\left\langle P^{2} \sigma_{ \pm, z}\right\rangle & =\left\langle P^{2}\right\rangle\left\langle\sigma_{ \pm, z}\right\rangle \\
\left\langle(P X+X P) \sigma_{ \pm, z}\right\rangle & =\langle(P X+X P)\rangle\left\langle\sigma_{ \pm, z}\right\rangle .
\end{aligned}
$$

As we are concerned with the long-time limit, we can calculate $\left\langle\sigma_{ \pm, z}\right\rangle$ in the stationary state using the optical Bloch equation for a two-level ion at rest at the (fixed) position $X=0$. The resulting equations for both traveling- and standing-wave configurations are listed in Appendix B. They can be summarized in matrix form as follows:

$$
\dot{\mathbf{X}}=B \mathbf{X}+2 C \mathbf{X} \cos (\omega t)+\mathbf{D},
$$

where $B$ and $C$ and $\mathbf{D}$ are constant $14 \times 14$ matrices and a constant vector, respectively, which can be readily read off from the equations of Appendix B. The whole set of dynamical variables has been written in a column vector $\mathbf{X}$, its components being the following expectation values: $x_{1}=\left\langle P^{2}\right\rangle, x_{2}=\left\langle X^{2}\right\rangle, x_{3}=\langle P X+X P\rangle$, etc. (see Appendix B).

Making the ansatz

$$
\mathbf{X}=\sum_{n=-\infty}^{\infty} \mathbf{Y}^{n} e^{i n \omega t}
$$

inserting it into (5.2), and identifying terms with the same time behavior we find the relation between different coefficients

$$
i n \omega \mathbf{Y}^{n}=B \mathbf{Y}^{n}+C\left(\mathbf{Y}^{n+1}+\mathbf{Y}^{n-1}\right)+\mathbf{D} \delta_{n, 0} .
$$

The solution to this equation can be given in terms of matrix continued fractions as

$$
\mathbf{Y}^{n+1}=H^{n} \mathbf{Y}^{n}
$$

where

$$
H^{n}=-\left[B-i \omega(n+1)+C H^{n+1}\right]^{-1} C
$$


and

$$
\mathbf{Y}^{0}=-\left[B+C\left(H^{0}+H^{0 *}\right)\right]^{-1} \mathbf{D}
$$

These formulas may be used to evaluate numerically $\left\langle P^{2}\right\rangle$ and $\left\langle X^{2}\right\rangle$, by taking $H^{n_{0}}=0$ for a given $n_{0}$ and checking that the results do not vary when $n_{0}$ is increased. We have verified that this method converges very rapidly for the first stable region in the $a$ - $q$ parameter space. In the unstable region unphysical results are found, such as negative kinetic energies, because in that case, the ion is heated by the trapping potential.

In the following we will show that some of the properties of the ion motion in the stationary state can be calculated without the necessity of evaluating (5.7). Once the ion has reached the stationary state due to laser cooling, one can try to ignore the presence of the laser-ion interaction between the external and internal degrees of freedom. This amounts to keeping only zeroth order terms in the evolution equations, which then take the form

$$
\begin{aligned}
& \dot{x}_{1}=-W x_{3}, \\
& \dot{x}_{2}=x_{3}, \\
& \dot{x}_{3}=2 x_{1}-2 W x_{2} .
\end{aligned}
$$

Again, making an ansatz for $x_{i}(i=1,2,3)$,

$$
x_{i}=\sum_{n=-\infty}^{\infty} y_{i}^{n} e^{i n \omega t}
$$

and proceeding as before we find the following relations:

$$
\begin{aligned}
\frac{\omega^{2}}{4} q\left(y_{3}^{n+1}+y_{3}^{n-1}\right) & =i \omega y_{1}^{n}+\frac{\omega^{2}}{4} a y_{3}^{n}, \\
0 & =i \omega n y_{2}^{n}-y_{3}^{n} \\
\frac{\omega^{2}}{4} q\left(y_{2}^{n+1}+y_{2}^{n-1}\right) & =-y_{1}^{n}+\frac{\omega^{2}}{4} a y_{2}^{n}+i \frac{\omega}{2} y_{3}^{n} .
\end{aligned}
$$

This set of equations can be easily solved. Indeed, the second and third equations directly give $y_{1}^{n}$ and $y_{3}^{n}$ in terms of $y_{2}^{n}$; a suitable linear combination of them all gives a relation between the $y_{2}^{n}$ variables:

$$
q\left[(2 n+1) y_{2}^{n+1}+(2 n-1) y_{2}^{n-1}\right]=2 n\left(a-n^{2}\right) y_{2}^{n}
$$

The solution to this equation can be given in a continued fraction form

$$
y_{2}^{n+1}=h^{n} y_{2}^{n}
$$

where

$$
h^{n}=\frac{q(2 n+1)}{2(n+1)\left[a-(n+1)^{2}\right]-q(2 n+3) h^{n+1}} .
$$

These formulas for $y_{2}^{n}$ are similar to (5.5) and (5.6), but now the variable $y_{2}^{0}$ remains undetermined. Hence Eqs. (5.8) do not have a unique solution; rather, the general solution is determined up to an overall factor $y_{2}^{0}$. This was expected, since the stationary state assumed in the Fourier expansion (5.9) is determined by cooling. It is the laser-ion interaction that determines the value of $y_{2}^{0}$ [starting from the solution (5.7)]. An analogous behavior is also found for a harmonic trap, since in that case the final energy can only be determined when the laser-ion interaction is taken into account. As one can show using simple power counting arguments, Eq. (5.12) can be used to find the remaining coefficients $y_{i}^{n}(i=1,2,3)$ starting from $y_{2}^{0}$, since the corrections given when the laser-ion interaction is included are of higher order in the LambDicke expansion. These coefficients are proportional to $y_{2}^{0}$ and consequently we can establish relationships between these coefficients without any need to consider the cooling process, i.e., using only (5.12).

The conclusion is that in order to study many features of the behavior of the trapped ion in the stationary state, one only has to deal with the Mathieu equation which, in terms of the appropriate variables, takes the form (5.8). For example, the ratio between the amplitude of the oscillation of the kinetic energy due to the micromotion and the mean kinetic energy depends only on the parameters that determine the micromotion, i.e., those appearing in the Mathieu equation ( $a$ and $q$ ). It is given by

$$
\frac{\left\langle P^{2}\right\rangle_{\max }-\left\langle P^{2}\right\rangle_{\min }}{\overline{\left\langle P^{2}\right\rangle}}=\frac{4 y_{1}^{1}}{y_{1}^{0}} \text {. }
$$

The expressions for $y_{1}^{1}$ and $y_{1}^{0}$ can be easily found from Eqs. (5.10). We find

$$
\frac{\left\langle P^{2}\right\rangle_{\max }-\left\langle P^{2}\right\rangle_{\min }}{\overline{\left\langle P^{2}\right\rangle}}=4 \frac{(a-2) h^{0}-q-q h^{1} h^{0}}{a-2 q h^{0}}
$$

where $h^{0}$ and $h^{1}$ are given by Eq. (5.13). In the particular case $a=0$ (which is the most often used in the experiments), this expression takes the simple form

$$
\frac{\left\langle P^{2}\right\rangle_{\max }-\left\langle P^{2}\right\rangle_{\min }}{\overline{\left\langle P^{2}\right\rangle}}=\frac{3}{4} q+\frac{25}{1152} q^{3}+o\left(q^{5}\right),
$$

which has proved to be in good agreement with the results obtained from the numerical solution of the full problem. In a similar way one can obtain other ratios such as $\left(\left\langle X^{2}\right\rangle_{\max }-\left\langle X^{2}\right\rangle_{\min }\right) / \overline{\left\langle X^{2}\right\rangle}$. However, if the final averaged kinetic energy is required, one must evaluate the matrix continued fractions (5.7) numerically, or, alternatively, use other qualitative arguments such as those given in the previous section.

\section{DISCUSSION}

In the preceding sections we have shown that the final mean kinetic energy, as given by $\overline{\left\langle P^{2}\right\rangle} / 2 m$, can indeed be calculated taking into account the micromotion in the Paul trap. Also, it was argued that we can understand the occurrence of the various minima and maxima of the final residual kinetic energy as arising from the interaction of the ion with several laser frequencies in a frame moving with the ion. As an illustration of this concept Fig. 5 shows $\overline{\left\langle P^{2}\right\rangle}$ as a function of the detuning for an ion localized at the node of a standing wave. Figures 

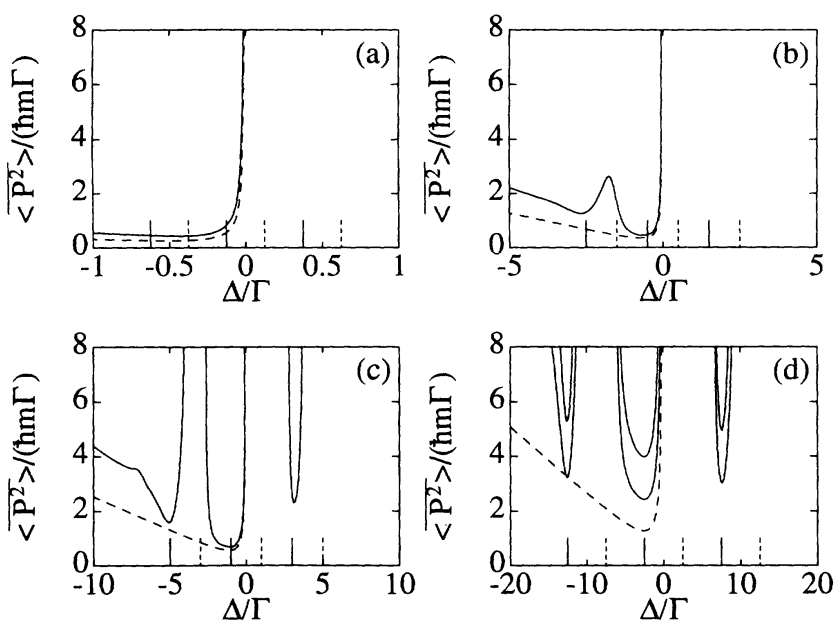

FIG. 5. Mean kinetic energy as a function of the detuning $\Delta$ for an ion at the node of a standing wave. $a=0$ and (a) $\omega=0.5 \Gamma$; (b) $\omega=2 \Gamma$; (c) $\omega=4 \Gamma$, and (d) $\omega=10 \Gamma$. In all the figures the ratio $\nu / \omega=1 / 4$. In (d) instead of the mean kinetic energy we have plotted the maximum and minimum values of the kinetic energy in one micromotion period. The dashed lines show the results calculated with a harmonic trap.

5(a)-5(d) show the mean kinetic energy for the same stability parameters $a$ and $q$, i.e., for a constant ratio $\nu / \omega=1 / 4$, with $\omega=0.5,2,4,10 \Gamma$ in Figs. 5(a) $-5(\mathrm{~d})$, respectively (under the Lamb-Dicke approximation, the results at the node of a standing wave do not depend on the Rabi frequency $\Omega$, nor on other parameters such as $k$ ). The dashed lines always show the expectation values in the harmonic potential approximation.

According to the concept outlined above (cf. discussion of Fig. 4), the small vertical lines indicate the position of the six different sideband frequencies contributing to the cooling-heating processes. Note in Fig. 5(a), the frequency $\omega$ is small so that the lasers interacting with the ion in its rest frame are close enough together to all interact with it, the strongest component $\left(\right.$ at $\left.\omega_{L}+\nu\right)$ prevails, and the minimum of the kinetic energy appears at $\Delta=-\Gamma / 2$ which corresponds to the optimum detuning for weak confinement (for $\omega=0.5, \nu=0.125$, and then $\Gamma>\nu)$. Note also that the mean kinetic energy is about twice the value expected for the harmonic trap case, as was stated in Sec. II.

In Fig. 5(b) the micromotion frequency and the trap frequency are further increased, i.e., in the rest frame of the ion the laser frequencies become more separated. Accordingly, cooling is observed for the detuning $-(\nu+\omega)$ [see the local dip in Fig. 5(b)], whereas for the detuning $\nu-\omega$ heating can be observed as indicated by the local maximum in Fig. 5(b). Increasing $\omega$ and $\nu$ further results in even greater separation of the frequencies [see Figs. 5(c) and 5(d)] and accordingly regions where cooling and heating appears are more and more separated and well resolved.

Cooling regions appear in the figures for detunings close to $-\nu,-(\nu+\omega)$, and $-(\nu-\omega)$, in agreement with the arguments presented above. Moreover, all the local minima in each of these regions have the same values, which coincide with that given by (4.11).

As has been argued in Sec. IV above, the greater the separation of the frequencies "sensed" by the ion, the better the ion can be considered as interacting only with a single frequency chosen by the given detuning in each case. This concept can be generalized to more levels, taking into account different separated resonances and provided that the laser frequencies "sensed" by the ion are well separated as, e.g., given by the inequality (4.5). As soon as more than one frequency contributes essentially to the cooling-heating process this concept is no longer applicable and the full and involved treatment, as outlined in Sec. V, has to be invoked in order to determine the exact values for the final kinetic energy.

As already mentioned in Sec. II, in the long-time limit (i.e., $t \rightarrow \infty$ ) the kinetic energy $\left\langle P(t)^{2}\right\rangle$ and the spatial variance $\left\langle X(t)^{2}\right\rangle$ are explicit functions of time. Their respective maximum values and minimum values were evaluated and given in Fig. 1. For a comparison with the mean values which are obtained by averaging over one period of the frequency $\omega$, the minimum and maximum values $\left\langle P^{2}\right\rangle_{\max }$ and $\left\langle P^{2}\right\rangle_{\min }$ are shown in Fig. 5(d). In many experiments the kinetic energy limits the achievable accuracy as, e.g., in time and frequency standard applications where the second order Doppler shift causes a major uncertainty. Thus, a residual micromotion actually leads to higher uncertainties than those expected for a harmonic trap. This problem has been addressed previously by Wineland et al. [15], who state that the energy contribution of the micromotion energy is equal to the kinetic energy of the secular motion as discussed above in Sec. II. However, they do not consider the time dependence of the additional micromotion energy which would lead to unwanted uncertainties in measurements. We find from Eq. (5.16) that the uncertainty in the kinetic energy can be given by the simple formula

$$
\frac{\left\langle P^{2}\right\rangle_{\max }-\left\langle P^{2}\right\rangle_{\min }}{\overline{\left\langle P^{2}\right\rangle}} \approx \frac{3}{4} q
$$

which is valid for all $q$ parameters in the stability region and for $a=0$. An expression which is valid in the whole stability region is given in (5.15). This uncertainty is to be compared with the quantum uncertainty which is determined by the final quantum state of the laser cooled ion. For sideband cooling as well as for Doppler cooling, using the results for the effective harmonic potential, we find that the quantum uncertainty is given by

$$
\frac{\Delta P^{2}}{\left\langle P^{2}\right\rangle}=\sqrt{2} \text {. }
$$

Hence, since always $3 q / 4<\sqrt{2}$ (in the first stability region), the uncertainty imposed by the residual micromotion is always smaller than the quantum uncertainty, though its contribution is negligible only for small values of $q$.

So far, all calculations have been carried out for cooling of a single ion placed at the node of a standing-wave laser field. As has been shown earlier [10] this leads to temperatures that are lower than for a traveling wave and 
independent of the applied Rabi frequency. Figure 3(a) shows a calculation of the mean kinetic energy as a function of the detuning for an ion located at the point of the maximum gradient in a standing wave (solid line). The parameters were $\Omega=5 \Gamma, \nu=1.5, \omega=10$, and the dashed line indicates the calculation for the harmonic trap case. Comparing this figure with Fig. 4(d) shows that the behavior is very similar to the case where the ion is localized at the node of a standing wave, except for the fact that now cooling is observed for blue detunings which is to be expected [10]. This is completely different for the case of traveling waves as is shown in Fig. 3(b). Here, the dashed line again indicates the expected behavior for a harmonic trap whereas the solid line shows the influence of the micromotion. In contrast to what is observed for a harmonic trap potential, the most striking result is that cooling is possible for blue detunings, i.e., for a detuning close to $\delta=\omega-\nu$. Note also that cooling near $\delta=-\nu$ is less efficient and for $\delta=-(\nu+\omega)$ is more efficient than expected from a harmonic trap.

For an experimental verification of the influence of the micromotion on laser cooling of an ion in a Paul trap we thus propose to investigate the cooling with traveling waves and for blue detunings. Observation of cooling as predicted by the calculations indicated in Fig. 3(b) would clearly demonstrate that the micromotion not only influences the cooling-heating behavior but indeed can be exploited for laser cooling of trapped ions in Paul traps. The condition (4.5) of course requires that $\nu>\Gamma$, however, this could be experimentally achieved either with narrow transitions as, e.g., in $\mathrm{In}^{+}$ions, or by new miniaturized trap designs.

\section{CONCLUSION}

In this paper we have studied theoretically laser cooling of a single trapped ion in a Paul trap with particular emphasis on the influence of the micromotion. Mean kinetic energies have been derived from the time averaged expectation value $\overline{\left\langle P^{2}\right\rangle}$ and compared with results obtained for a purely harmonic trap. The results show that for the strong-binding limit the micromotion leads to a new and unexpected cooling behavior whereas in the weakbinding limit the micromotion increases the residual energies but does not change the cooling dynamics given by the harmonic trap approximation. The theoretical results presented here open the prospect for further, more detailed, understanding of laser cooling in Paul traps and it is expected that in new trap designs the micromotion can be used for additional cooling.

\section{ACKNOWLEDGMENTS}

J.I.C. and R.B. acknowledge travel support from NATO. R.B. is supported in part by the Deutsche Forschungsgemeinschaft. L.J.G. is supported in part by DGCYT under Contract No. PB-0052 and by a Basque Country Grant. He also thanks the Instituto de Optica, CSIC, for its hospitality. The work at JILA is supported in part by the National Science Foundation. The ESA
IUE Observatory is affiliated with the Astrophysics Division, Space Science Department, ESTEC.

\section{APPENDIX A: QUANTUM MATHIEU EQUATION}

In this appendix we analyze in detail the quantum version of the Mathieu equation and derive some results used in Sec. IV. Starting from Hamiltonian (2.1), the position operator $X(t)$ in the Heisenberg picture (interaction picture of Sec. IV) satisfies the Mathieu equation

$$
\ddot{X}(t)+\frac{\omega^{2}}{4}[a-2 q \cos (\omega t)] X(t)=0 .
$$

In view of the linearity of this equation we make the ansatz

$$
X(t)=\frac{1}{\sqrt{2} \xi}\left[A^{\dagger} F(t)+A F(t)^{*}\right],
$$

where $A$ and $A^{\dagger}$ are now (time-independent) operators related to the initial condition at $t=0$. Substituting this in the Mathieu equation, one finds that $F(t)$ is that given by the ( $c$ number) function (2.5) provided the parameter $\xi$ is defined as

$$
\xi=|F(0) \dot{F}(0)|^{1 / 2}
$$

The operators $A$ and $A^{\dagger}$ are linear combinations of the initial position and momentum operators, $X(0)=X$ and $P(0)=P$, respectively. It can be readily checked that they satisfy the commutation relation $\left[A, A^{\dagger}\right]=1$. Taking the time derivative of (A2) we find that for the operator $P(t)$ (we have taken $m=1$ )

$$
P(t)=\frac{1}{\sqrt{2} \xi}\left[A^{\dagger} \dot{F}(t)+A \dot{F}(t)^{*}\right]
$$

Starting from (A2) and (A4), we have for the expectation values of the operators $X^{2}$ and $P^{2}$

$$
\begin{aligned}
\left\langle X(t)^{2}\right\rangle= & \operatorname{Tr}\left[X(t)^{2} \rho\right] \\
= & \frac{1}{2 \xi^{2}}\left\{|F(t)|^{2} \operatorname{Tr}\left[\left(A^{\dagger} A+A^{\dagger} A\right) \rho\right]\right. \\
& \left.+F(t)^{2} \operatorname{Tr}\left[A^{\dagger 2} \rho\right]+F(t)^{* 2} \operatorname{Tr}\left[A^{2} \rho\right]\right\}
\end{aligned}
$$

$$
\begin{aligned}
\left\langle P(t)^{2}\right\rangle= & \operatorname{Tr}\left[P(t)^{2} \rho\right] \\
= & \frac{1}{2 \xi^{2}}\left\{|\dot{F}(t)|^{2} \operatorname{Tr}\left[\left(A^{\dagger} A+A^{\dagger} A\right) \rho\right]\right. \\
& \left.-\dot{F}(t)^{2} \operatorname{Tr}\left[A^{\dagger 2} \rho\right]-\dot{F}(t)^{* 2} \operatorname{Tr}\left[A^{2} \rho\right]\right\}
\end{aligned}
$$

Finally, we can define a basis in the Hilbert space for 
the external degrees of freedom through

$$
A^{\dagger} A|N\rangle=N|N\rangle
$$

so that $A$ and $A^{\dagger}$ can be regarded as annihilation and creation operators in this Fock basis. Expressing the density operator $\rho$ in this new basis one can readily calculate the expectation value of any external observable.

\section{APPENDIX B: EVOLUTION EQUATIONS FOR STANDING- AND TRAVELING-WAVE LASER CONFIGURATIONS}

In this appendix we list the evolution equations for an ion in a Paul trap up to second order in the Lamb-Dicke expansion. In order to do that we define $\left(x_{1}, x_{2}, \ldots, x_{14}\right)=\mathbf{X}^{T}$ by

$$
\mathbf{X}^{T}=\left(\left\langle P^{2}\right\rangle,\left\langle X^{2}\right\rangle,\langle X P+P X\rangle,\left\langle X \sigma_{x}\right\rangle,\left\langle X \sigma_{y}\right\rangle,\left\langle X \sigma_{z}\right\rangle,\left\langle P \sigma_{x}\right\rangle,\left\langle P \sigma_{y}\right\rangle,\left\langle P \sigma_{z}\right\rangle,\langle X\rangle,\langle P\rangle,\left\langle\sigma_{x}\right\rangle,\left\langle\sigma_{y}\right\rangle,\left\langle\sigma_{z}\right\rangle\right)
$$

For a standing-wave configuration these equations read

$$
\begin{aligned}
\dot{x}_{1} & =-\left(W+\Omega_{c}\left\langle\sigma_{x}\right\rangle_{\mathrm{SS}}\right) x_{3}-\Omega_{a} x_{7}+\frac{\Gamma}{2} \alpha k^{2}\left(1+\left\langle\sigma_{z}\right\rangle_{\mathrm{SS}}\right) \\
\dot{x}_{2} & =x_{3} \\
\dot{x}_{3} & =2 x_{1}-2\left(W+\Omega_{c}\left\langle\sigma_{x}\right\rangle_{\mathrm{SS}}\right) x_{2}-\Omega_{a} x_{4} \\
\dot{x}_{4} & =-\gamma x_{4}+\Delta x_{5}+x_{7} \\
\dot{x}_{5} & =-\Omega_{a}\left\langle\sigma_{z}\right\rangle_{\mathrm{SS}} x_{2}-\Delta x_{4}-\gamma x_{5}-\Omega_{\varphi} x_{6}+x_{8} \\
\dot{x}_{6} & =\Omega_{a}\left\langle\sigma_{y}\right\rangle_{\mathrm{SS}} x_{2}+\Omega_{\varphi} x_{5}-2 \gamma x_{6}+x_{9}-2 \gamma x_{10} \\
\dot{x}_{7} & =-W x_{4}-\gamma x_{7}+\Delta x_{8}-\frac{\Omega_{a}}{2} \\
\dot{x}_{8} & =-\frac{\Omega_{a}}{2}\left\langle\sigma_{z}\right\rangle_{\mathrm{SS}} x_{3}-W x_{5}-\Delta x_{7}-\gamma x_{8}-\Omega_{\varphi} x_{9} \\
\dot{x}_{9} & =\frac{\Omega_{a}}{2}\left\langle\sigma_{y}\right\rangle_{\mathrm{SS}} x_{3}-W x_{6}+\Omega_{\varphi} x_{8}-2 \gamma x_{9}-2 \gamma x_{11} \\
\dot{x}_{10} & =x_{11}, \\
\dot{x}_{11} & =-W x_{10}-\frac{\Omega_{a}}{2} x_{12} \\
\dot{x}_{12} & =-\gamma x_{12}+\Delta x_{13} \\
\dot{x}_{13} & =-\Omega_{a} x_{6}-\Delta x_{12}-\gamma x_{1} 3-\Omega_{\varphi} x_{14} \\
\dot{x}_{14} & =\Omega_{a} x_{5}+\Omega_{\varphi} x_{13}-2 \gamma x_{14}-2 \gamma
\end{aligned}
$$

where $\gamma=\Gamma / 2, \Omega_{\varphi}=\Omega \cos \phi, \Omega_{a}=-k \Omega \sin \phi$, and $\Omega_{c}=-k^{2} \Omega_{\varphi} / 2$.

For traveling-wave configurations, we have

$$
\begin{aligned}
& \dot{x}_{1}=-\left(W+\Omega_{c}\left\langle\sigma_{x}\right\rangle_{\mathrm{SS}}\right) x_{3}-\Omega_{b} x_{8}+\frac{\Gamma}{2} \alpha k^{2}\left(1+\left\langle\sigma_{z}\right\rangle_{\mathrm{SS}}\right), \\
& \dot{x}_{2}=x_{3} \\
& \dot{x}_{3}=2 x_{1}-2\left(W+\Omega_{c}\left\langle\sigma_{x}\right\rangle_{\mathrm{SS}}\right) x_{2}-\Omega_{b} x_{5} \\
& \dot{x}_{4}=-\gamma x_{4}+\Delta x_{5}+x_{7}+\Omega_{b}\left\langle\sigma_{z}\right\rangle_{\mathrm{SS}} x_{2}
\end{aligned}
$$

$$
\begin{aligned}
\dot{x}_{5} & =-\Delta x_{4}-\gamma x_{5}-\Omega_{\varphi} x_{6}+x_{8} \\
\dot{x}_{6} & =\Omega_{b}\left\langle\sigma_{x}\right\rangle_{\mathrm{SS}} x_{2}+\Omega_{\varphi} x_{5}-2 \gamma x_{6}+x_{9}-2 \gamma x_{10} \\
\dot{x}_{7} & =\frac{\Omega_{b}}{2}\left\langle\sigma_{z}\right\rangle_{\mathrm{SS}} x_{3}-W x_{4}-\gamma x_{7}+\Delta x_{8} \\
\dot{x}_{8} & =-W x_{5}-\Delta x_{7}-\gamma x_{8}-\Omega_{\varphi} x_{9}-\frac{\Omega_{b}}{2} \\
\dot{x}_{9} & =\frac{\Omega_{b}}{2}\left\langle\sigma_{x}\right\rangle_{\mathrm{SS}} x_{3}-W x_{6}+\Omega_{\varphi} x_{8}-2 \gamma x_{9}-2 \gamma x_{11} \\
\dot{x}_{10} & =x_{11} \\
\dot{x}_{11} & =-W x_{10}-\frac{\Omega_{b}}{2} x_{13} \\
\dot{x}_{12} & =-\gamma x_{12}+\Delta x_{13}+\Omega_{b} x_{6} \\
\dot{x}_{13} & =-\Delta x_{12}-\gamma x_{1} 3-\Omega_{\varphi} x_{14} \\
\dot{x}_{14} & =\Omega_{b} x_{4}+\Omega_{\varphi} x_{13}-2 \gamma x_{14}-2 \gamma
\end{aligned}
$$

where $\Omega_{b}=k \Omega$ and $\Omega_{c}=-k^{2} \Omega / 2$. In all these equations $\left\langle\sigma_{x}\right\rangle_{\mathrm{SS}},\left\langle\sigma_{y}\right\rangle_{\mathrm{SS}},\left\langle\sigma_{z}\right\rangle_{\mathrm{SS}}$ are the components of the Bloch vector in steady state, derived for a two-level atom at rest at the position $X=0[10]$.

Note that a simple power counting performed on these evolution equations shows that the solutions $x_{1,2,3}$ have zeroth order contributions while the others are of the first order at least. In addition, looking at the equations for $\dot{x}_{4, \ldots, 14}$ one can readily see that only the zeroth order of $x_{1,2,3}$ is necessary and that by no means do these equations determine it. After an analysis, there seem to be more variables than equations. However this is not the case. The choice of an appropriate cutoff $\left(n_{0} \rightarrow \infty\right)$ to calculate the values of $y_{i}^{n}\left(n=0, \pm 1, \ldots, \pm n_{0}\right)$ produces the additional equations to determine the problem. In the numerical calculation in terms of matrix continued fractions (see Sec. V), a finite $n_{0}$ substitutes for the infinite limit. Note that this is also true in the harmonic trap case, being in this case $n_{0}=0$.
[1] D. J. Wineland, W. M. Itano, and R. S. VanDyck, Jr., Adv. At. Mol. Phys. 19, 135 (1983).

[2] See, e.g., J. Mod. Opt. 39, 192 (1992).

[3] P. E. Toschek, in New Trends in Atomic Physics Vol. I, Proceedings of the Les Houches Summer School, Session XVIII, edited by G. Grynberg and R. Stora (NorthHolland, Amsterdam, 1984), p. 381.

[4] R. Blatt, in Fundamental Systems in Quantum Optics, Proceedings of the Les Houches Summer School, Session LIII, edited by J. Dalibard, J. M. Raymond, and J. Zinn-
Justin (North-Holland, Amsterdam, 1993) p. 253.

[5] D. J. Wineland and W. M. Itano, Phys. Rev. A 20, 1521 (1979); W. M. Itano and D. J. Wineland, ibid. 25, 35 (1982).

[6] S. Stenholm, Rev. Mod. Phys. 58, 699 (1986).

[7] M. Lindberg and S. Stenholm, J. Phys. B 17, 3375 (1985).

[8] M. Lindberg and J. Javanainen, J. Opt. Soc. Am. B 3, 1008 (1986).

[9] Th. Sauter, R. Blatt, W. Neuhauser, and P. E. Toschek, 
Phys. Scr. T22, 216 (1988).

[10] J. I. Cirac, R. Blatt, P. Zoller, and W. D. Phillips, Phys. Rev. A 46, 2668 (1992).

[11] R. Blatt, P. Zoller, G. Holzmüller, and I. Siemers, Z. Phys. D 4, 121 (1986).

[12] E. Fischer, Z. Phys. 156, 1 (1959).

[13] The form of the coefficients $c_{2 n}$ for the Mathieu equation in some limiting cases, as well as some properties of this equation, can be found, for example, in M. Abramowitz and I. A. Stegun, Handbook of Mathematical Functions
(Dover, New York, 1964). Note that in order to fix these coefficients we have chosen $c_{0}=1$.

[14] R. J. Glauber, in Foundations of Quantum Mechanics, edited by T. D. Black, M. M. Nieto, H. S. Pilloff, M. O. Scully, and R. M. Sinclair (World Scientific, Singapore, 1992), p. 23.

[15] D. J. Wineland, W. M. Itano, J. C. Bergquist, and R. G. Hulet, Phys. Rev. A 36, 2220 (1987).

[16] R. J. Cook, D. G. Shankland, and A. L. Wells, Phys. Rev. A 31, 564 (1985). 